PROCEEDINGS OF THE

AMERICAN MATHEMATICAL SOCIETY

Volume 127, Number 7, Pages 1957-1966

S 0002-9939(99)04761-9

Article electronically published on February 26, 1999

\title{
MEASURES ON FINITE CONCRETE LOGICS
}

\author{
PETER G. OVCHINNIKOV
}

(Communicated by Andreas R. Blass)

\begin{abstract}
We examine the possibility to extend measures and signed measures on a concrete logic on a finite set to those on all its subsets.
\end{abstract}

\section{INTRODUCTION}

In this paper, we study the possibility to extend measures and signed measures on a concrete logic consisting of subsets of a finite set to those on the Boolean algebra of all its subsets. Of course, the case when the finite concrete logic in question is a Boolean algebra is trivial and requires no theory. General concrete logics on finite sets may possess a very complex structure. Measures and signed measures on them may behave extremely nontrivially and unexpectedly. This area has remained little-studied thus far, though some special finite concrete logics were examined by a number of investigators (cf. [11], [22], [24], [26], [29], [30]).

Orthomodular posets (OMPs) (cf. [1], [9], [13], [22], [27], [28]) were introduced as a generalization of the so-called logical approach to the foundations of quantum mechanics initiated by G. Birkhoff and J. von Neumann [3]. Sub-OMPs of the Boolean algebra of all subsets of a set are called concrete logics. As all OMPs can, concrete logics can serve as domains for measures and signed measures. This has resulted in the creation of the so-called generalized measure theory [10] generalizing the classical measure theory on $\sigma$-algebras. Finite concrete logics provide a new specific subject for a combinatorial and measure-theoretical investigation.

A general approach to extending measures and signed measures on finite concrete logics based on a dual description for the latter was suggested by the author in [25]. Here, we develop the approach. Along with the aforementioned study of some particular finite concrete logics, it is the additivity of integrals for observables on concrete logics (cf. [7], [10], [18], [21], [33]) that essentially stimulates one to do this. Also, it is noteworthy that the well-known G. Birkhoff theorem on doubly stochastic matrices admits a formulation in terms of measures on a suitable finite concrete logic (see [22], [25]). The theory of measures on projections (see, e.g., [4], $[5],[8],[14]-[16],[31],[32])$, as a model, has considerably influenced this research, too.

Received by the editors August 15, 1996 and, in revised form, October 8, 1997.

1991 Mathematics Subject Classification. Primary 06C15; Secondary 81P10.

Key words and phrases. Finite concrete logic, measure, signed measure.

This research was supported by the Russian Foundation for Fundamental Research, grants no. 95-01-00025 and no. 96-01-01265. 
In Section 2, we recall the basic definitions and some concepts, results, and notation of [25].

In Section 3, we quote a result by J. Zerbe and S. Gudder on the additivity of integrals, and then we introduce and handle the concrete logics of the form $\Delta(\Lambda)$ yielded by finite point collections on the plane. We prove that all concrete logics $\Delta(\{0, \ldots, n-1\} \times\{0, \ldots, m-1\})$ are regular.

In Section 4, we introduce new concepts of the local positivity and the filledness of a finite concrete logic and establish theorems on them. In particular, this enables us to negatively solve the long-standing problem of whether every $\Delta(\Lambda)$ is positive.

Section 5 is dedicated to an algebraic generalization of the concept of closedness. The symmetric (i.e., closed with respect to the symmetric difference) finite concrete logics are characterized. One of the characterizations is accomplished in terms of the well-known combinatorial game Nim.

In Section 6, we pose several open questions.

\section{Preliminaries}

Let $\Gamma$ be a set. We denote by $\mathcal{P}(\Gamma)$ the Boolean algebra of all subsets of $\Gamma$. A concrete logic [9], [10], [27], [28] on $\Gamma$ is an $\mathcal{E} \subset \mathcal{P}(\Gamma)$ satisfying

1) $\Gamma \in \mathcal{E}$

2) $x \in \mathcal{E} \Longrightarrow \Gamma \backslash x \in \mathcal{E}$

3) $x, y \in \mathcal{E}, x \cap y=\emptyset \Longrightarrow x \cup y \in \mathcal{E}$.

Let $\mathcal{E}$ be a concrete logic on $\Gamma$. A signed measure on $\mathcal{E}$ is a mapping $\mu: \mathcal{E} \rightarrow \mathbb{R}$ such that

$$
x, y \in \mathcal{E}, x \cap y=\emptyset \Longrightarrow \mu(x \cup y)=\mu(x)+\mu(y) .
$$

Let $V(\mathcal{E})$ denote the real vector space of all signed measures on $\mathcal{E}$. A signed measure $\mu$ on $\mathcal{E}$ is called a measure on $\mathcal{E}$ provided that $\mu(x) \geq 0$ for all $x \in \mathcal{E}$. A state on $\mathcal{E}$ is a measure $\mu$ on $\mathcal{E}$ with $\mu(\Gamma)=1$. A state $\mu$ on $\mathcal{E}$ is called two-valued if $\mu(\mathcal{E})=\{0,1\}$. Let $S(\mathcal{E})$ and $S^{0,1}(\mathcal{E})$ be the sets of all states and all two-valued states on $\mathcal{E}$, respectively. For an arbitrary $\omega \in \Gamma$ put

$$
\mu_{\omega}(x)=\left\{\begin{array}{ll}
0 & \text { if } \omega \notin x, \\
1 & \text { if } \omega \in x
\end{array} \quad(x \in \mathcal{E}) .\right.
$$

Obviously $\mu_{\omega} \in S^{0,1}(\mathcal{E})$. The $\mu_{\omega}$ is called the point state defined by $\omega$. Denote $S^{\text {point }}(\mathcal{E})=\left\{\mu_{\omega} \mid \omega \in \Gamma\right\}$. A state $\mu$ on $\mathcal{E}$ is called pure if $\mu$ is an extreme point of the convex set $S(\mathcal{E})$. Let $S^{\text {pure }}(\mathcal{E})$ denote the set of all pure states on $\mathcal{E}$. Every minimal element of $\mathcal{E} \backslash\{\emptyset\}$ with respect to inclusion is called an atom in $\mathcal{E}$. Let $\alpha(\mathcal{E})$ denote the set of all atoms in $\mathcal{E}$.

Next, let $\Omega$ be a finite set. Denote by $E$ the set of all concrete logics on $\Omega$.

Obviously every $\mathcal{E} \in E$ coincides with the set of all disjoint unions of elements of $\alpha(\mathcal{E})$.

Let $A \subset \mathcal{P}(\Omega) \backslash\{\emptyset\}$. It is easy to see that the following conditions are equivalent:

(i) there exists $\mathcal{E} \in E$ with $\alpha(\mathcal{E})=A$;

(ii) with respect to inclusion, the elements of $A$ are mutually noncomparable, and for every $A_{1} \subset A$ with pairwise disjoint elements there exists $A_{2} \subset A$ with pairwise disjoint elements satisfying

$$
\Omega \backslash\left(\bigcup_{x \in A_{1}} x\right)=\bigcup_{x \in A_{2}} x .
$$


Let $\mathcal{E} \in E$.

Definition 2.1 ([25]). $\mathcal{E}$ is called regular if every signed measure on $\mathcal{E}$ extends to a signed measure on $\mathcal{P}(\Omega)$.

Definition $2.2([25])$. $\mathcal{E}$ is called positive if every measure on $\mathcal{E}$ which extends to a signed measure on $\mathcal{P}(\Omega)$ also extends to a measure on $\mathcal{P}(\Omega)$.

Let $R$ and $P$ denote the sets of all regular and all positive concrete logics on $\Omega$, respectively.

Obviously every measure on $\mathcal{E}$ extends to a measure on $\mathcal{P}(\Omega)$ if and only if $\mathcal{E} \in R \cap P$. (It is clear that if every measure on $\mathcal{E}$ extends to a signed measure on $\mathcal{P}(\Omega)$, then $\mathcal{E} \in R$ as every signed measure on $\mathcal{E}$ is a difference of two measures on $\mathcal{E}$.) Also,

$$
\mathcal{E} \in R \cap P \Longleftrightarrow S^{\text {pure }}(\mathcal{E})=S^{\text {point }}(\mathcal{E}) .
$$

Next, we put $\mathcal{M}=\{\mu \in V(\mathcal{P}(\Omega)) \mid \mu(\Omega)=0\}$. Obviously $\mathcal{M}$ is a linear subspace of the finite-dimensional vector space $V(\mathcal{P}(\Omega))$. For every $S \subset \mathcal{M}$ and $T \subset \mathcal{P}(\Omega)$ put

$$
S^{\circ}=\{x \in \mathcal{P}(\Omega) \mid \mu(x)=0 \text { for all } \mu \in S\}
$$

and

$$
T^{\circ}=\{\mu \in \mathcal{M} \mid \mu(x)=0 \text { for all } x \in T\} .
$$

Obviously $S^{\circ} \in E$ for every $S \subset \mathcal{M}$, and $T^{\circ}$ is a linear subspace of $\mathcal{M}$ for every $T \subset$ $\mathcal{P}(\Omega)$. The couple of mappings ${ }^{\circ}: \mathcal{P}(\mathcal{M}) \rightarrow \mathcal{P}(\mathcal{P}(\Omega))$ and ${ }^{\circ}: \mathcal{P}(\mathcal{P}(\Omega)) \rightarrow \mathcal{P}(\mathcal{M})$ (we hope them to not be confused), $\mathcal{P}(\mathcal{M})$ and $\mathcal{P}(\mathcal{P}(\Omega))$ being ordered by the inclusion is a Galois correspondence (see [2], [6], [25]). $S \in \mathcal{P}(\mathcal{M})(T \in \mathcal{P}(\mathcal{P}(\Omega))$ ) is called closed [25] if $S^{\circ \circ}=S\left(T^{\circ \circ}=T\right)$. Let $M$ and $N$ be the sets of all closed elements of $\mathcal{P}(\mathcal{M})$ and $\mathcal{P}(\mathcal{P}(\Omega))$, respectively. We have $N \subset E$. It follows from well-known general properties of Galois correspondences (see, e.g., [2], [6]) that $M$ and $N$ ordered by the inclusion are finite lattices, and the mappings ${ }^{\circ}: M \rightarrow N$ and ${ }^{\circ}: N \rightarrow M$ are mutually inverse antiisomorphisms between them.

Theorem 2.3 ([25]). For every $\mathcal{E} \in N$ there exists $\mu \in \mathcal{M}$ with $\mathcal{E}=\{\mu\}^{\circ}$.

Remark 2.4. In Theorem 2.3, $\mu$ may be chosen $\mathbb{Z}$-valued.

Theorem 2.5 ([25]). Let $\mathcal{E} \in E$. Then the following three conditions are equivalent:

1) $\mathcal{E} \in R$;

2) $\operatorname{dim} \mathcal{E}^{\circ}+\operatorname{dim} V(\mathcal{E}) \leq \operatorname{card} \Omega$;

3) $\operatorname{dim} \mathcal{E}^{\circ}+\operatorname{dim} V(\mathcal{E})=\operatorname{card} \Omega$.

Theorem 2.6 ([25]). $P \subset N$.

Remark 2.7. It was also shown in [25] that $N \not \subset P$, and $R$ is noncomparable, with respect to inclusion, with $P$ or $N$ even in case $\operatorname{card} \Omega=6$.

We conclude the section with giving an elementary example aimed to initially demonstrate the strength of the above methods.

Let $n \in \mathbb{N}$ and $\Omega_{n}=\{0,1\}^{n}$. For every $i \in\{1, \ldots, n\}$ put $o_{i}=\left\{x \in \Omega_{n} \mid x_{i}=0\right\}$ and $t_{i}=\left\{x \in \Omega_{n} \mid x_{i}=1\right\}$. Also set $\mathcal{E}_{n}=\left\{\emptyset, \Omega_{n}, o_{1}, \ldots, o_{n}, t_{1}, \ldots, t_{n}\right\}$. Obviously $\mathcal{E}_{n}$ is a concrete logic on $\Omega_{n}$. (It is easy to see that $\mathcal{E}_{n}$, as an OMP, is isomorphic 
to $M O n$ (cf. [13]).) Let us solve the following two problems: 1) prove that $\mathcal{E}_{n}$ is closed for every $n \in \mathbb{N} ; 2)$ for every $n \in \mathbb{N}$, calculate $\operatorname{dim} \mathcal{E}_{n}^{\circ}$.

Let us consider the affine bijection $\Phi: \mu \mapsto\left(\mu\left(o_{1}\right), \ldots, \mu\left(o_{n}\right)\right)$ between $S\left(\mathcal{E}_{n}\right)$ and $[0,1]^{n}$. Since $\Phi\left(S^{0,1}\left(\mathcal{E}_{n}\right)\right)=\{0,1\}^{n}$, it follows that $S^{\text {pure }}\left(\mathcal{E}_{n}\right)=S^{0,1}\left(\mathcal{E}_{n}\right)$. Obviously $S^{0,1}\left(\mathcal{E}_{n}\right)=S^{\text {point }}\left(\mathcal{E}_{n}\right)$. This implies that $\mathcal{E}_{n}$ is regular and positive. By Theorem 2.6, $\mathcal{E}_{n}$ is closed. By Theorem 2.5, we obtain $\operatorname{dim} \mathcal{E}_{n}^{\circ}=\operatorname{card} \Omega_{n}-\operatorname{dim} V\left(\mathcal{E}_{n}\right)=$ $2^{n}-n-1$.

\section{CONCRETE LOGICS OF THE FORM $\Delta(\Lambda)$}

In this section, we first recall a remarkable Zerbe-Gudder theorem.

Let $\Pi$ be a (maybe, infinite) set and $\Sigma$ be a concrete logic on $\Pi$ which is a $\sigma$-class [9], [10], [27], [28], i.e., for every sequence $\left(x_{n}\right)$ of elements of $\Sigma$ with $x_{i} \cap x_{j}=\emptyset$ $(i \neq j)$ it holds $\bigcup_{n=1}^{\infty} x_{n} \in \Sigma$. Let $\mu$ be a signed measure on $\Sigma$ which is $\sigma$-additive, i.e., for every sequence $\left(x_{n}\right)$ of elements of $\Sigma$ satisfying $x_{i} \cap x_{j}=\emptyset(i \neq j)$ one has $\mu\left(\bigcup_{n=1}^{\infty} x_{n}\right)=\sum_{n=1}^{\infty} \mu\left(x_{n}\right)$. A function $f: \Pi \rightarrow \mathbb{R}$ is called $\Sigma$-measurable if $f^{-1}(A) \in \Sigma$ for all Borel $A \subset \mathbb{R}$. If $f: \Pi \rightarrow \mathbb{R}$ is a bounded $\Sigma$-measurable function, then we may consider the Gudder integral $\int f d \mu$ [9], [10] defined as follows:

1) $\Sigma_{f}=\left\{f^{-1}(A) \mid A \subset \mathbb{R}\right.$ is Borel $\}$ is a $\sigma$-algebra on $\Pi$;

2) $\mu_{f}=\mu \mid \Sigma_{f}$ is a $\sigma$-additive signed measure on $\Sigma_{f}$;

3) $f$ is a bounded $\Sigma_{f}$-measurable function;

4) there exists the Lebesgue integral $\int f d \mu_{f}$;

5) define $\int f d \mu=\int f d \mu_{f}$.

Theorem 3.1 ([33], also cf. [18]). Let $f, g: \Pi \rightarrow \mathbb{R}$ be finitely valued and such that $f, g$, and $f+g$ are $\Sigma$-measurable. Then $\int(f+g) d \mu=\int f d \mu+\int g d \mu$.

Remark 3.2. Theorem 3.1 does not hold for arbitrary bounded functions $f, g$ such that $f, g$, and $f+g$ are $\Sigma$-measurable (see [21]). Still, the requirement that $f$ and $g$ are finitely valued may be weakened (cf. [18]). The additivity of the Gudder integral can also fail for unbounded functions (cf. [7]).

Of course, all finite concrete logics are $\sigma$-classes, and all signed measures on them are $\sigma$-additive. Now, let us introduce an interesting particular class of finite concrete logics closely related to the above theorem. Let $\Lambda \subset \mathbb{R}^{2}$ be finite. Denote by $\Delta(\Lambda)$ the least, with respect to inclusion, concrete logic on $\Lambda$ such that $\pi_{1}, \pi_{2}$, and $\pi_{1}+\pi_{2}$ are $\Delta(\Lambda)$-measurable, wherein $\pi_{1}(x, y)=x, \pi_{2}(x, y)=y((x, y) \in \Lambda)$.

Theorem 3.3. $\Delta(\Lambda)$, where $\Lambda=\{0, \ldots, n-1\} \times\{0, \ldots, m-1\}$ is regular for all $n, m \in \mathbb{N}$.

For each $i \in\{0, \ldots, n-1\}, j \in\{0, \ldots, m-1\}$, and $k \in\{0, \ldots, n+m-2\}$ put $C_{i}=\pi_{1}^{-1}(\{i\}), R_{j}=\pi_{2}^{-1}(\{j\})$, and $D_{k}=\left(\pi_{1}+\pi_{2}\right)^{-1}(\{k\})$. We start with two lemmas.

Lemma 3.4. Suppose that $n, m \geq 3$. Let $\mu \in \Delta(\Lambda)^{\circ}$ satisfy $\mu(\{\omega\})=0$ for all $\omega \in\{0, \ldots, n-3\} \times\{2, \ldots, m-1\}$. Then $\mu=0$.

Proof. As $D_{0} \in \Delta(\Lambda)$, we have $\mu(\{(0,0)\})=0$. As $C_{0} \in \Delta(\Lambda)$, we have $\mu(\{(0,1)\})=$ 0 . As $D_{1} \in \Delta(\Lambda)$, we have $\mu(\{(1,0)\})=0$. As $C_{1} \in \Delta(\Lambda)$, we have $\mu(\{(1,1)\})=0$, etc. Thus $\mu(\{\omega\})=0$ for all $\omega \in(\{0, \ldots, n-3\} \times\{0,1\}) \cup\{(n-2,0)\}$. Analogously, $\mu(\{\omega\})=0$ for all $\omega \in(\{n-2, n-1\} \times\{2, \ldots, m-1\}) \cup\{(n-1,1)\}$. As $R_{1} \in \Delta(\Lambda)$, we get $\mu(\{(n-2,1)\})=0$. Since $R_{0} \in \Delta(\Lambda)$, we eventually obtain $\mu(\{(n-1,0)\})=0$. 
Lemma 3.5. Suppose that $n+m \geq 3$. Let $\mu \in V(\Delta(\Lambda))$ satisfy $\mu\left(C_{i}\right)=\mu\left(R_{j}\right)=$ $\mu\left(D_{k}\right)=0$ for all $i \in\{1, \ldots, n-1\}, j \in\{1, \ldots, m-1\}$, and $k \in\{0,2, \ldots, n+$ $m-2\}$. Then $\mu=0$.

Proof. It suffices to show that $\mu\left(C_{0}\right)=\mu\left(R_{0}\right)=\mu\left(D_{1}\right)=0$. By Theorem 3.1, $\int\left(\pi_{1}+\pi_{2}\right) d \mu=\int \pi_{1} d \mu+\int \pi_{2} d \mu$. This means that

$$
\sum_{k=1}^{n+m-2} k \mu\left(D_{k}\right)=\sum_{i=1}^{n-1} i \mu\left(C_{i}\right)+\sum_{j=1}^{m-1} j \mu\left(R_{j}\right) .
$$

Hence $\mu\left(D_{1}\right)=0$. Since obviously $\sum_{i=0}^{n-1} \mu\left(C_{i}\right)=\sum_{k=0}^{n+m-2} \mu\left(D_{k}\right)$, it follows that $\mu\left(C_{0}\right)=0$. Since $\sum_{j=0}^{m-1} \mu\left(R_{j}\right)=\sum_{k=0}^{n+m-2} \mu\left(D_{k}\right)$, we have $\mu\left(R_{0}\right)=0$.

Proof of Theorem 3.3. If $n<3$ or $m<3$, then obviously $\Delta(\Lambda)=\mathcal{P}(\Lambda)$, and the theorem follows. Suppose that $n, m \geq 3$. By Lemma 3.4, $\operatorname{dim} \Delta(\Lambda)^{\circ} \leq$ $(n-2)(m-2)$. By Lemma 3.5, $\operatorname{dim} V(\Delta(\Lambda)) \leq 2 n+2 m-4$. Therefore, $\operatorname{dim} \Delta(\Lambda)^{\circ}+$ $\operatorname{dim} V(\Delta(\Lambda)) \leq n m=\operatorname{card} \Lambda$. By Theorem 2.5, $\Delta(\Lambda)$ is regular.

Remark 3.6. It follows from [21] that $S^{0,1}(\Delta(\Lambda))=S^{\text {point }}(\Delta(\Lambda))$ for every finite $\Lambda \subset \mathbb{R}^{2}$.

\section{LOCAL POSITIVITY AND FILLEDNESS}

Let $\Omega$ be a finite set again, and let $\mathcal{E}$ be a concrete logic on $\Omega$.

Definition 4.1. A set $x \subset \Omega$ is called $\mathcal{E}$-strange if $y \not \subset x$ for all $y \in \mathcal{E} \backslash\{\emptyset\}$. Let $\operatorname{St}(\mathcal{E})$ denote the set of all $\mathcal{E}$-strange subsets of $\Omega$.

Let us give two examples of how $\mathcal{E}$-strange sets can arise.

Example 4.2. $\alpha\left(\mathcal{E}^{\circ \circ}\right) \backslash \mathcal{E} \subset \operatorname{St}(\mathcal{E})$.

Example 4.3. $x \backslash\{\omega\} \in \operatorname{St}(\mathcal{E})$ for all $x \in \alpha(\mathcal{E})$ and $\omega \in x$.

Definition 4.4. $\mathcal{E}$ is called locally positive if for every $x \in \operatorname{St}(\mathcal{E})$ there exists $\mu \in \mathcal{E}^{\circ}$ satisfying $\mu(\{\omega\})>0$ for all $\omega \in x$.

Remark 4.5. In Definition 4.4, we could take the set of all maximal, with respect to inclusion, elements of $\operatorname{St}(\mathcal{E})$ instead of $\operatorname{St}(\mathcal{E})$, and we would come to an equivalent definition.

Theorem 4.6. If $\mathcal{E}$ is positive, then $\mathcal{E}$ is locally positive.

Proof. Let $x \in \operatorname{St}(\mathcal{E})$. Define $\nu \in V(\mathcal{P}(\Omega))$ by

$$
\nu(\{\omega\})= \begin{cases}-1 & \text { if } \omega \in x, \\ \operatorname{card} x & \text { if } \omega \in \Omega \backslash x .\end{cases}
$$

Obviously $\nu \mid \mathcal{E}$ is a measure on $\mathcal{E}$. Since $\mathcal{E}$ is positive, there exists $\mu \in \mathcal{E}^{\circ}$ such that $\nu+\mu$ is a measure on $\mathcal{P}(\Omega)$. For every $\omega \in x$ we have $-1+\mu(\{\omega\})=(\nu+\mu)(\{\omega\}) \geq 0$. Thus $\mu(\{\omega\}) \geq 1>0$.

Remark 4.7. By Example 4.2, if $\mathcal{E}$ is locally positive, then $\mathcal{E}$ is closed. By Theorem 4.6, this generalizes Theorem 2.6. 
Now, let us apply Theorem 4.6 to concrete logics of the form $\Delta(\Lambda)$. Put $\Lambda=$ $\{0,1,2,3\}^{2}$. Let us prove that $\Delta(\Lambda)$ is not positive.

It is easy to verify that $\Delta(\Lambda)^{\circ}=\{\mu(a, b, c, d) \mid a, b, c, d \in \mathbb{R}\}$, where

$\mu(a, b, c, d)=$\begin{tabular}{|c|c|c|c|}
\hline$a$ & $b$ & $-a-b$ & 0 \\
\hline$c$ & $d$ & $\begin{array}{c}-a-b \\
-c-d\end{array}$ & $a+b$ \\
\hline$-a-c$ & $-a-b$ & $\begin{array}{c}a+b \\
\end{array}$ & $\begin{array}{c}a+c \\
+c-d\end{array}$ \\
\hline 0 & $a+c$ & $\begin{array}{c}a+b \\
+d\end{array}$ & $-2 a-b$ \\
& & $+c-d$ \\
\hline
\end{tabular}

in the sense that we identify squares with their centers and inscribe into them the values of the signed measure $\mu(a, b, c, d)$ on the corresponding singletons. Put $x=(\{0,1\} \times\{2,3\}) \cup\{(3,0)\}$. Then $x \in \operatorname{St}(\Delta(\Lambda))$ as $x$ contains no nonempty subsets with identically zero sums of the values. At the same time, there exists no $\mu \in \Delta(\Lambda)^{\circ}$ with $\mu(\{\omega\})>0$ for all $\omega \in x$ as $a, b, c, d$, and $-2 a-b-c-d$ cannot simultaneously be positive. Thus $\Delta(\Lambda)$ is not locally positive. By Theorem 4.6, $\Delta(\Lambda)$ is not positive.

This example answers a question posed by the author in 1989 and then discussed during the Second Winter School on Measure Theory at Liptovský Ján.

As F. Sultanbekov observed, $\Delta(\{0, \ldots, n-1\} \times\{0, \ldots, m-1\})$ is similarly not locally positive whenever $n \geq 4$ and $m \geq 4$.

Definition 4.8. Let $x \subset \Omega$. The polar rank of $x$ with respect to $\mathcal{E}, \operatorname{pr}_{\mathcal{E}}(x)$, is defined as $\operatorname{pr}_{\mathcal{E}}(x)=\operatorname{dim} V_{x}$, where $V_{x}=\left\{\mu|\mathcal{P}(x)| \mu \in \mathcal{E}^{\circ}\right\}$.

So, $\operatorname{pr}_{\mathcal{E}}(x) \leq \min \left(\operatorname{dim} \mathcal{E}^{\circ}, \operatorname{card} x\right)$ by definition. Obviously $\operatorname{pr}_{\mathcal{E}}(x) \leq(\operatorname{card} x)-1$ whenever $x \in \mathcal{E}^{\circ \circ}$.

Definition 4.9. $\mathcal{E}$ is called filled if $\operatorname{pr}_{\mathcal{E}}(x)=(\operatorname{card} x)-1$ for every $x \in \alpha(\mathcal{E})$.

Obviously if $\mathcal{E}$ is filled, then $\operatorname{card} x \leq\left(\operatorname{dim} \mathcal{E}^{\circ}\right)+1$ for all $x \in \alpha(\mathcal{E})$.

Theorem 4.10. $\mathcal{E}$ is filled if and only if for every $x \in \alpha(\mathcal{E})$ and every $\omega \in x$ there exists $\mu \in \mathcal{E}^{\circ}$ satisfying $\mu(\{\tau\})>0$ for all $\tau \in x \backslash\{\omega\}$.

Lemma 4.11. Let $e_{1}, \ldots, e_{n} \in \mathbb{R}^{n}$ be such that $\sum_{j=1}^{n}\left(e_{i}\right)_{j}<0$ for all $i \in\{1, \ldots, n\}$ and $\left(e_{i}\right)_{j} \geq 0$ for all $i \neq j$. Then $e_{1}, \ldots, e_{n}$ are linearly independent.

Proof. Let $A$ be a matrix whose rows are $e_{1}, \ldots, e_{n}$. Let $f_{1}, \ldots, f_{n}$ be the columns of $A$. It suffices to prove that $f_{1}, \ldots, f_{n}$ are linearly independent. Suppose on the contrary that $\sum_{i=1}^{n} \lambda_{i} f_{i}=0$, where not all $\lambda_{i} \in \mathbb{R}$ equal 0 . We may assume that $0<\lambda_{1}=\max _{1 \leq i \leq n}\left|\lambda_{i}\right|$. Then we have $0=\sum_{i=1}^{n} \lambda_{i}\left(f_{i}\right)_{1}=\sum_{i=1}^{n} \lambda_{i}\left(e_{1}\right)_{i} \leq$ $\lambda_{1} \sum_{i=1}^{n}\left(e_{1}\right)_{i}<0$. This is a contradiction.

Remark 4.12. Lemma 4.11 is also immediate from the Levy-Desplanques theorem (cf. [12]).

Proof of Theorem 4.10. The "only if" part is obvious.

"If." Let $x \in \alpha(\mathcal{E})$. Take an arbitrary $\omega \in x$. Let $x \backslash\{\omega\}=\left\{\omega_{1}, \ldots, \omega_{n}\right\}$, $\omega_{1}, \ldots, \omega_{n}$ being pairwise distinct. For each $i \in\{1, \ldots, n\}$ let $\mu_{i} \in \mathcal{E}^{\circ}$ satisfy $\mu_{i}(\{\tau\})>0$ for all $\tau \in x \backslash\left\{\omega_{i}\right\}$. Put $e_{i}=\left(\mu_{i}\left(\left\{\omega_{1}\right\}\right), \ldots, \mu_{i}\left(\left\{\omega_{n}\right\}\right)\right)(i \in\{1, \ldots, n\})$. For every $i \in\{1, \ldots, n\}$ we have $\sum_{j=1}^{n}\left(e_{i}\right)_{j}=\sum_{j=1}^{n} \mu_{i}\left(\left\{\omega_{j}\right\}\right)=-\mu_{i}(\{\omega\})<0$. For 
all $i \neq j$ we obtain $\left(e_{i}\right)_{j}=\mu_{i}\left(\left\{\omega_{j}\right\}\right)>0$. By Lemma $4.11, e_{1}, \ldots, e_{n}$ are linearly independent. This obviously means that $\operatorname{pr}_{\mathcal{E}}(x)=n=(\operatorname{card} x)-1$.

Remark 4.13. In the proof of Theorem 4.10, we do not utilize that $\exists \mu \in \mathcal{E}^{\circ} \forall \tau \in$ $x \backslash\{\omega\}(\mu(\{\tau\})>0)$. This means that if $x \in \alpha(\mathcal{E}), \omega \in x$, and $\forall \delta \in x \backslash\{\omega\} \exists \mu \in$ $\mathcal{E}^{\circ} \forall \tau \in x \backslash\{\delta\}(\mu(\{\tau\})>0)$, then $\exists \mu \in \mathcal{E}^{\circ} \forall \tau \in x \backslash\{\omega\}(\mu(\{\tau\})>0)$.

Corollary 4.14. If $\mathcal{E}$ is locally positive, then $\mathcal{E}$ is filled.

Corollary 4.15. If $\mathcal{E}$ is locally positive, then $\operatorname{card} x \leq\left(\operatorname{dim} \mathcal{E}^{\circ}\right)+1$ for all $x \in \alpha(\mathcal{E})$.

Remark 4.16. For all that $\Delta\left(\{0,1,2,3\}^{2}\right)$ is not locally positive, it is closed and filled. Also, it is easy to give examples showing that the closedness and the filledness of $\mathcal{E}$ do not entail each other even in case card $\Omega=6$.

\section{A generalization: The abstract Closedness. Symmetric Finite CONCRETE LOGICS}

As in Section 4, $\Omega$ stands for a finite set, and $\mathcal{E}$ denotes a concrete logic on $\Omega$.

Definition 5.1. Let $G$ be an abelian group. $\mathcal{E}$ is called $G$-abstractly closed if there exists a mapping $F: \Omega \rightarrow G$ with $\mathcal{E}=\left\{x \subset \Omega \mid \sum_{\omega \in x} F(\omega)=0\right\}$.

Remark 5.2. By Theorem $2.3, \mathcal{E}$ is closed just in case $\mathcal{E}$ is $(\mathbb{R},+)$-abstractly closed. Also, the concrete logic on $\{1,2,3,4\}$ formed by the subsets with even numbers of elements is not closed, but is $\left(\mathbb{Z}_{2},+\right)$-abstractly closed, $\mathbb{Z}_{2}$ being the two-element field. Thus the $G$-abstract closedness for some abelian group $G$ does not imply the closedness. It is easy to see that if $G$ is an abelian group, then $\mathcal{E}$ is $G$-abstractly closed if and only if $\mathcal{E}$ is the kernel (cf. [17], [19], [20]) of some $G$-valued measure $\mu$ on $\mathcal{P}(\Omega)$ with $\mu(\Omega)=0$.

Remark 5.3. If we take an abelian group $G$ and a mapping $F: \Omega \rightarrow G$ with $\sum_{\Omega}$. $F(\omega)=0$, then $\left\{x \subset \Omega \mid \sum_{\omega \in x} F(\omega)=0\right\}$ is obviously a concrete logic on

Proposition 5.4. The following two conditions are equivalent:

$1)$ there exists an abelian group $G$ such that $\mathcal{E}$ is $G$-abstractly closed;

2) if $x \subset \Omega$ is such that for every abelian group $G$ and every mapping $F: \Omega \rightarrow G$ with $\sum_{\omega \in y} F(\omega)=0$ for all $y \in \mathcal{E}$ it holds $\sum_{\omega \in x} F(\omega)=0$, then $x \in \mathcal{E}$.

Proof. Obviously 1) $\Longrightarrow 2$ ).

$2) \Longrightarrow 1$ ). For each $x \in \mathcal{P}(\Omega) \backslash \mathcal{E}$ let $G_{x}$ be an abelian group and $F_{x}: \Omega \rightarrow G_{x}$ be a mapping such that $\sum_{\omega \in y} F_{x}(\omega)=0$ for all $y \in \mathcal{E}$, and $\sum_{\omega \in x} F_{x}(\omega) \neq 0$. Consider the direct sum $G=\bigoplus_{x \in \mathcal{P}(\Omega) \backslash \mathcal{E}} G_{x}$ and the mapping $F: \omega \mapsto\left(F_{x}(\omega)\right)_{x \in \mathcal{P}(\Omega) \backslash \mathcal{E}}$ from $\Omega$ to $G$. It is easy to verify that $\mathcal{E}=\left\{x \subset \Omega \mid \sum_{\omega \in x} F(\omega)=0\right\}$. Thus $\mathcal{E}$ is $G$-abstractly closed.

Remark 5.5. If $\left(\mathcal{E}_{i}\right)_{i \in I}$ is a family of concrete logics on $\Omega$ such that for every $i \in I$, $\mathcal{E}_{i}$ is $G_{i}$-abstractly closed, $G_{i}$ being an abelian group, then $\bigcap_{i \in I} \mathcal{E}_{i}$ is obviously a $\prod_{i \in I} G_{i}$-abstractly closed concrete logic on $\Omega$.

Let us present an example of a concrete logic on a finite set which is not $G$ abstractly closed for any abelian group $G$. (Another such example can be found in [20].) 
Example 5.6. Put $H=\{x \subset\{1, \ldots, 6\} \mid \operatorname{card} x \equiv 0(\bmod 3), x \neq\{1,2,3\}$, and $x \neq\{4,5,6\}\}$. Then $H$ is a concrete logic on $\{1, \ldots, 6\}$. Let $G$ be an abelian group. Suppose that $F:\{1, \ldots, 6\} \rightarrow G$ satisfies $H=\left\{x \subset\{1, \ldots, 6\} \mid \sum_{\omega \in x} F(\omega)=0\right\}$. As $\{1,4,5\},\{2,4,5\} \in H$, we get $F(1)=F(2)$. Similarly, $F(2)=F(3)$ and $F(4)=$ $F(5)=F(6)$. Let $a, b \in G$ satisfy $F(1)=F(2)=F(3)=a$ and $F(4)=F(5)=$ $F(6)=b$. Since $\{1,2,4\},\{1,4,5\} \in H$, we have $2 a+b=0=a+2 b$, whence $a=b$ and $3 a=0$. Since $F(1)+F(2)+F(3)=3 a=0$, it follows that $\{1,2,3\} \in H$. This is a contradiction.

Definition 5.7. $\mathcal{E}$ is called symmetric provided that $x, y \in \mathcal{E} \Longrightarrow x+y \in \mathcal{E},+$ being the symmetric difference.

Remark 5.8. If $\mathcal{E}$ is symmetric, then $\mathcal{E}$ can be viewed as a binary code.

Next, denote by $\mathbb{Z}_{2}^{\infty}$ the direct sum of countably many copies of $\left(\mathbb{Z}_{2},+\right.$ ). (Thus $\mathbb{Z}_{2}^{\infty}$ is the set of all sequences of zeros and ones with finite sets of the ones endowed with the structure of an abelian group, the addition being modulo 2 in every component.)

Let us now recall the rules of the Nim. Two players participate. There are several heaps, and each heap contains several things. By a move, a player chooses a heap and takes away an arbitrary number of things from that heap only, at least one and perhaps all. The players alternate their moves. The player who makes the last move wins.

A position, in the Nim, is the corresponding finite set of heaps. Let $I$ and $I I$ be the players, and let $I$ begin. Let $\Pi$ be a fixed position in which $I I$ possesses a winning strategy. Denote with $\mathcal{L}(\Pi)$ the set of all $x \subset \Pi$ such that $I I$ has a winning strategy in the position $x$. Let us show that $\mathcal{L}(\Pi)$ is a concrete logic on $\Pi$. We will do this without making use of the well-known description of the winning strategies in the Nim. 1) By definition, $\Pi \in \mathcal{L}(\Pi)$. 2) Let $x \in \mathcal{L}(\Pi)$. Suppose that $\Pi \backslash x \notin \mathcal{L}(\Pi)$. Then $I$ has a winning strategy in $\Pi \backslash x$. Let us show that $I$ has a winning strategy in $\Pi$, and this will be a contradiction. Let $I$ begin in accordance with his winning strategy in $\Pi \backslash x$ and separately play in $x$ or $\Pi \backslash x$ using winning strategies for $I I$ or $I$, respectively. Clear that $I$ will win. 3) Let $x, y \in \mathcal{L}(\Pi)$ satisfy $x \cap y=\emptyset$. Then $x \cup y \in \mathcal{L}(\Pi)$ as $I I$ can separately play in $x$ or $y$ according to winning strategies for $I I$.

Recall that two concrete logics, $\mathcal{E}_{1}$ on a set $\Omega_{1}$ and $\mathcal{E}_{2}$ on a set $\Omega_{2}$, are referred to as isomorphic ones if there exists a bijection $\varphi: \Omega_{1} \rightarrow \Omega_{2}$ satisfying $x \in \mathcal{E}_{1} \Longleftrightarrow$ $\varphi(x) \in \mathcal{E}_{2}$ for every $x \subset \Omega_{1}$.

Theorem 5.9. The following three conditions are equivalent:

1) $\mathcal{E}$ is symmetric;

2) $\mathcal{E}$ is $\mathbb{Z}_{2}^{\infty}$-abstractly closed;

3) there exists a position $\Pi$ in the Nim such that II has a winning strategy in $\Pi$ and $\mathcal{E}$ is isomorphic to $\mathcal{L}(\Pi)$.

Proof. 1$) \Longrightarrow 2)$. $(\mathcal{P}(\Omega),+)$ is a vector space over $\mathbb{Z}_{2} . \mathcal{E}$ is a linear subspace of $\mathcal{P}(\Omega)$. Thus there exist linear functionals $f_{1}, \ldots, f_{n}$ on $\mathcal{P}(\Omega)$ with $\mathcal{E}=\bigcap_{i=1}^{n} \operatorname{Ker} f_{i}$. Define a mapping $F: \Omega \rightarrow \mathbb{Z}_{2}^{\infty}$ as $F(\omega)=\left(f_{1}(\{\omega\}), \ldots, f_{n}(\{\omega\}), 0, \ldots\right)(\omega \in \Omega)$. Obviously $\mathcal{E}=\left\{x \subset \Omega \mid \sum_{\omega \in x} F(\omega)=0\right\}$.

The implication 2) $\Longrightarrow 1$ ) is obvious. It follows from the generally well-known description of the winning strategies in the Nim that 2$) \Longleftrightarrow 3$ ). 


\section{Open QUESTIONS}

Problem $6.1([23])$. Does $V(\mathcal{E})=\operatorname{Lin} S^{0,1}(\mathcal{E})$ hold for every concrete logic $\mathcal{E}$ on a finite set?

Problem 6.2. Is every $\Delta(\Lambda), \Lambda \subset \mathbb{R}^{2}$ being finite a) regular? b) closed? c) filled?

Problem 6.3. Is every locally positive concrete logic on a finite set positive?

Problem 6.4. Which concrete logics on finite sets can be represented with combinatorial games similarly to symmetric ones?

Problem 6.5. Give a direct "playing" proof that $\mathcal{L}(\Pi)$ is symmetric.

\section{ACKNOWLEDGMENTS}

The author is indebted to D. Mushtari, M. Navara, and the members of the Kazan seminar on "Algebras of Operators and Their Applications" and F. Sultanbekov especially for fruitful conversations.

\section{REFERENCES}

1. L. Beran, Orthomodular lattices. Algebraic approach, Academia, Prague, 1984. MR 86m:06015a

2. G. Birkhoff, Lattice theory, AMS, Providence, RI, 1967. MR 37:2638

3. G. Birkhoff and J. von Neumann, The logic of quantum mechanics, Ann. of Math. 37 (1936), 823-843.

4. L. Bunce and J. Maitland Wright, The Mackey-Gleason problem, Bull. Amer. Math. Soc. 26 (1992), 288-293. MR 92i:46070

5. E. Christensen, Measures on projections and physical states, Comm. Math. Phys. 86 (1982), 529-538. MR 85b:46072

6. P. Cohn, Universal algebra, Harper \& Row, New York, 1965. MR 31:224

7. J. Dravecký and J. Šipoš, On the additivity of Gudder integral, Math. Slovaca 30 (1980), 299-303. MR 82b:28009

8. A. Gleason, Measures on the closed subspaces of a Hilbert space, J. Math. Mech. 6 (1957), 885-893. MR 20:2609

9. S. Gudder, Stochastic methods in quantum mechanics, North Holland, New York, 1979.

10. _ An extension of classical measure theory, SIAM Rev. 26 (1984), 71-89. MR 85d:81014

11. S. Gudder and J.-P. Marchand, A coarse-grained measure theory, Bull. Acad. Pol. Sc. 28 (1980), 557-564. MR 82i:28004

12. R. Horn and C. Johnson, Matrix analysis, Cambridge University Press, Cambridge, 1986. MR 87e:15001

13. G. Kalmbach, Orthomodular lattices, Academic Press, London, 1983. MR 85f:06012

14. M. Matveichuk, A theorem on states on quantum logics, Teor. Mat. Fizika 45 (2) (1980), 244-250 (Russian). MR 82d:81015

15. _ A theorem on states on quantum logics, II, Teor. Mat. Fizika 48 (3) (1981), 261-265 (Russian). MR 83a:81004

16. (3) (1981), 41-53 (Russian).

17. R. Mayet and M. Navara, Classes of logics representable as kernels of measures, in Contr. General Algebra 9 (G.Pilz, ed.), Teubner, Stuttgart/Wien, 1995, pp. 241-248.

18. M. Navara, When is the integral on quantum probability spaces additive?, Real Anal. Exchange 14 (1989), 228-234. MR 90c: 81018

19. __ Kernel logics, Tatra Mountains Math. Publ. 3 (1993), 27-30. MR 95e:06024

20. _ Quantum logics representable as kernels of measures, Czechoslovak Math. J. 46 (1996), 587-597. MR 97j:81027

21. M. Navara and P. Pták, Two-valued measures on $\sigma$-classes, Čas. Pěst. Mat. 108 (1983), 225-229. MR 84m:28006 
22. P. Ovchinnikov, Structure of measures on quantum logics, Thesis, Kazan State University, Kazan, Russia, 1985 (Russian).

23. _ Problem 4, in Proc. Second Winter School on Measure Theory, Liptovský Ján, January 7-12, 1990, Czecho-Slovakia (A. Dvurečenskij and S. Pulmannová, eds.), p. 219.

24. Analiz (Kazan) 8 (1992), 95-98 (Russian). MR 94e:81020

25. __ A Galois correspondence connected with extending signed measures on a $\sigma$-class of subsets of a finite set, Izv. Vyssh. Uchebn. Zaved. Mat. (5) (1994), 36-40 (Russian). MR 95i: 81019

26. R. Prather, Generating the k-subsets of an n-set, Amer. Math. Monthly 87 (1980), 740-743.

27. P. Pták and S. Pulmannová, Orthomodular structures as quantum logics, Kluwer, Dordrecht, 1991. MR 82e:05005

28. A. Sherstnev, On Boolean logics, Uch. Zap. KGU 128 (1968), 48-62 (Russian).

29. F. Sultanbekov, Signed measures and automorphisms of a class of finite concrete logics, Konstr. Teoriya Funktsií i Funk. Analiz (Kazan) 8 (1992), 57-68 (Russian). MR 94f:81010

30. _ Set logics and their representations, Int. J. Theor. Phys. 32 (1993), 2177-2186. MR 95c:06019

31. F. Yeadon, Measures on projections in $W^{*}$-algebras of type $I I_{1}$, Bull. London Math. Soc. 15 (1983), 139-145. MR 84g:46089

32. $\quad$ Finitely additive measures on projections in finite $W^{*}$-algebras, Bull. London Math. Soc. 16 (1984), 145-150. MR 85i:46087

33. J. Zerbe and S. Gudder, Additivity of integrals on generalized measure spaces, J. Comb. Theory (A) 39 (1985), 42-52. MR 86m:81020

Department of Mathematics, Kazan State University, 420008, Kazan, Russia

E-mail address: Petr.Ovchinnikov@ksu.ru 